\title{
Moxibustion and other acupuncture point stimulation methods to treat breech presentation: a systematic review of clinical trials Xun $\mathrm{Li}^{1}$, Jun $\mathrm{Hu}^{2}$, Xiaoyi Wang ${ }^{3}$, Huirui Zhang ${ }^{3}$ and Jianping Liu*1,4
}

Address: ${ }^{1}$ Centre for Evidence-Based Chinese Medicine, Beijing University of Chinese Medicine, Beijing, PR China, ${ }^{2}$ Centre for the History of Medicine, Peking University, Beijing, PR China, ${ }^{3}$ School of Preclinical Medicine, Beijing University of Chinese Medicine, Beijing, PR China and ${ }^{4}$ National Research Centre in Complementary and Alternative Medicine (NAFKAM), University of Tromsø, Tromsø, Norway

Email: Xun Li - tina000341@163.com; Jun Hu - hujun@hsc.pku.edu.cn; Xiaoyi Wang - wangxiaoyi6666@sina.com; Huirui Zhang - tanghaohuizi@126.com; Jianping Liu* - Jianping.Liu@fagmed.uit.no

* Corresponding author

Published: 27 February 2009

Chinese Medicine 2009, 4:4 doi:10.1 I86/1749-8546-4-4

This article is available from: http://www.cmjournal.org/content/4/I/4

This is an Open Access article distributed under the terms of the Creative Commons Attribution License (http://creativecommons.org/licenses/by/2.0), which permits unrestricted use, distribution, and reproduction in any medium, provided the original work is properly cited.

\begin{abstract}
Background: Moxibustion, acupuncture and other acupoint stimulations are commonly used for the correction of breech presentation. This systematic review aims to evaluate the efficacy and safety of moxibustion and other acupoint stimulations to treat breech presentation.

Methods: We included randomized controlled trials (RCTs) and controlled clinical trials (CCTs) on moxibustion, acupuncture or any other acupoint stimulating methods for breech presentation in pregnant women. All searches in PubMed, the Cochrane Library (2008 Issue 2), China National Knowledge Information (CNKI), Chinese Scientific Journal Database (VIP) and WanFang Database ended in July 2008. Two authors extracted and analyzed the data independently.

Results: Ten RCTs involving 2090 participants and seven CCTs involving 1409 participants were included in the present study. Meta-analysis showed significant differences between moxibustion and no treatment (RR I.35, $95 \% \mathrm{Cl} \mathrm{I.20} \mathrm{to} \mathrm{I.5I;} 3$ RCTs). Comparison between moxibustion and knee-chest position did not show significant differences (RR I.30, $95 \% \mathrm{Cl} 0.95$ to I.79; 3 RCTs). Moxibustion plus other therapeutic methods showed significant beneficial effects (RR I.36, 95\% Cl I. $2 \mathrm{I}$ to I.54; 2 RCTs). Laser stimulation was more effective than assuming the knee-chest position plus pelvis rotating. Moxibustion was more effective than no treatment (RR I.29, $95 \% \mathrm{Cl} \mathrm{I.17}$ to I.42; 2 CCTs) but was not more effective than the knee-chest position treatment (RR I.22, 95\% $\mathrm{Cl}$ I.I I to I.34; 2 CCTs). Laser stimulation at Zhiyin (BL67) was more effective than the knee-chest position treatment (RR I.30, $95 \%$ Cl I.10 to I.54; 2 CCTs,).
\end{abstract}

Conclusion: Moxibustion, acupuncture and laser acupoint stimulation tend to be effective in the correction of breech presentation.

\section{Background}

Breech presentation (opposite direction of the normal position of the foetus) is common in the mid-trimester of pregnancy, with the incidence of breech decreasing as the pregnancy approaches term. The incidence of breech pres- entation at term is reported to be $4 \%$ [1]. Women with breech presentation face serious problems if delivering vaginally. Breech presentation may arise from placenta praevia, multiple gestation, uterine abnormalities, poor uterine tone, pre-maturity or unknown causes, and is 
associated with primigravidae, older mothers, babies that are small for gestational age and female babies [2].

Caesarean section is often recommended for pregnant women with breech presentation who may otherwise prefer natural deliveries. Caesarean section does have distinct disadvantages including increased risks of maternal urinary tract infection, haemorrhage, wound infection and scar dehiscence or uterine rupture during subsequent labour [3].

Some conventional non-surgical therapies for breech presentation are available, such as the knee-chest position treatment and external manual cephalic version. However, knee-chest position is difficult to adopt and likely to cause inadequate compliance, whereas external cephalic version is much more complex, potentially dangerous, time consuming and expensive [4].

Moxibustion is a traditional method of burning moxa sticks (usually made from herbal preparations containing Artemisia vulgaris) near an acupoint to cause a warm and painless sensation [5]. In China, moxibustion on Zhiyin (BL67) point has long been used to correct abnormal foetal position and is widely used to correct breech presentation in obstetrics. Many clinical studies on this method were carried out and published in academic journals including JAMA [6]. Moxibustion and other acupoint stimulation methods such as acupuncture and laser stimulation were found to be effective to treat breech presentation.

The present systematic review aims to evaluate the efficacy and safety of moxibustion and other acupoint stimulation methods to treat breech presentation in pregnant women.

\section{Methods}

\section{Databases and search strategy}

Two authors (JH and XL) searched the China National Knowledge Infrastructure (CNKI) (1979-2008), Chinese Scientific Journal Database (VIP) (1989-2008), WanFang Database (WanFang) for Chinese Publications (19852008), PubMed (1966-2008), the Cochrane Library (Issue 2, 2008) and Traditional Chinese Medicine Database System. The last search was in July 2008. The search terms included 'abnormal foetal position (taiwei buzheng)', 'breech presentation (tunwei)', 'correction/conversion (zhuantai)', 'correct abnormal foetal presentation (jiaozheng taiwei/jiuzheng taiwei)', 'moxibustion', 'acupuncture', 'pregnancy', 'acupoint stimulation', 'Zhiyin' and 'laser'. We manually retrieved some recognized articles not available electronically and also performed additional searches to identify potentially eligible trials from the retrieved studies and reviews in the electronic databases.

\section{Inclusion criteria}

We included randomized controlled trials (RCTs) and non-randomized controlled clinical trials (CCTs) on moxibustion and/or other acupoint stimulation methods. There was no restriction on the race or gestation of participants, publication type or language. We excluded casecontrol studies, case series, case reports, non-clinical studies and trials to compare different acupoint stimulation methods.

\section{Study selection and data extraction}

Three authors (XL, XYW and HRZ) selected studies according to the inclusion criteria. The paper titles and abstracts were read and assessed for their eligibility and relevance. The full texts of related papers were retrieved and reviewed based on the inclusion and exclusion criteria of the studies. We were not blinded to the names of the authors, institutions or journals of the published studies.

Two authors (XYW and HRZ) extracted the data independently using a structured data extraction form and another author (XL) verified the extracted data. Any discrepancies were discussed and consensus was reached. The extracted data included demographic data, quality of trial design, inclusion and exclusion criteria, interventions and results.

In the case of missing data in the included studies, we contacted the original investigators and conducted the intention to treat analysis (ITT).

\section{Quality assessment}

Three authors (XYW, HRZ and JH) assessed the quality of each trial independently, according to the Cochrane handbook [7] and CONSORT statement for reporting RCTs [8,9], A generic grading system [10] was applied to the included RCTs and CCTs as follows:

A (good)

Studies in this category have the least biases and their results are considered valid. These studies have (1) clear description of the population, setting, interventions and comparison groups; (2) appropriate measurement of outcomes; (3) appropriate statistical and analytical methods; (4) no reporting errors; (5) less than 20 percent dropouts; (6) clear reporting of dropouts; and (7) appropriate consideration and adjustment for potential confounders.

\section{$B$ (fair)}

Studies in this category are susceptible to some degrees of biases that are not sufficient to invalidate the results. These studies may have sub-optimal adjustments for potential confounders and may also lack certain information that is needed to assess limitations and potential problems. 


\section{$C$ (poor)}

Studies in this category have significant biases which may invalidate the results. These studies may have critical flaws in design, analysis and/or reporting, missing information and/or discrepancies in reporting. For instance, these studies either do not consider potential confounders or do not make adjustments for them appropriately.

The studies graded between $\mathrm{B}$ (fair) and $\mathrm{C}$ (poor) were reviewed and graded again by other authors. Consensus was reached through discussion. It should be noted that this summary quality grading system evaluates and grades the studies within their own design strata and does not attempt to assess the comparative validity of studies across different designs. Thus, the assessors and users should be cognizant of the study design when interpreting the methodological quality grade of a study.

\section{Data analysis}

Review Manager Software 4.2.7 provided by the Cochrane Collaboration was used for data analysis. Dichotomous data were expressed as a risk ratio (RR) with a provision of 95\% confidence interval (CI). Meta-analysis was performed if experimental intervention and control intervention were the same or similar, such as moxibustion versus knee-chest position. The statistical heterogeneity was analyzed and presented when I square $\left(\mathrm{I}^{2}\right)$ is over $50 \%$ or $P<$ 0.1 as significant. Random effect model was used for the meta-analysis if there was significant heterogeneity $\left(\mathrm{I}^{2}>\right.$ $50 \%$ ) and fixed effect model was used when the heterogeneity was not significant $\left(\mathrm{I}^{2}<50 \%\right)[11]$.

\section{Results \\ Description of included studies}

In this review, a total of 869 studies were screened out of which 148 studies with full texts were retrieved for selection according to the inclusion and exclusion criteria. A total of 131 papers were excluded, out of which 112 papers were duplicate publications, case reports, case series, review articles, basic research or mechanism studies, and 19 studies did not meet the inclusion criteria in terms of participants, interventions or outcomes (Additional file 1$)$. As a result, 17 clinical trials including ten RCTs [6,12-20] and seven CCTs [21-27] were reviewed (Figure 1). In addition, two systematic reviews were identified. One of them is a Cochrane review which covered three trials published in 2005 [28] and another review covered six trials published in 2008 [29]. Three trials were conducted in Italy $[14,15,24]$, one in Japan [22] and the remaining 13 in China. Six trials were published in English $[6,14,15,17,22,24]$ and three trials had more than two arms.

Among the included trials, ten RCTs and seven CCTs involved 2090 and 1409 pregnant women respectively. These trials investigated moxibustion (13 trials), acupunc- ture ( 2 trials), electro-acupuncture ( 1 trial), laser stimulation ( 2 trials) or ear acupuncture $(1$ trial) on acupoints and comparisons with no treatment ( 7 trials), knee-chest position (10 trials), raising buttocks method ( 2 trials) or throwing breech ( 1 trial) for the correction of breech presentation (Table 1). Among all the included trials, 14 trials $[6,12-19,21,22,24-26]$ used ultrasound to confirm the diagnosis of breech presentation. Four trials reported adverse effects.

\section{Methodological qualities}

Three RCTs $[6,14,15]$ published in English were rated as A (good). One RCT published in Chinese [12], which met the inclusion criteria, was rated as $\mathrm{C}$ (poor) and the remaining RCTs were rated as B (fair). One CCT [25], which met the inclusion criteria, was rated as $\mathrm{C}$ (poor) and the remaining CCTs were all rated as B (fair).

\section{Efficacy of the interventions}

Moxibustion and/or acupuncture on Zhiyin versus no treatment Three RCTs $[6,14,20]$ found significant differences between moxibustion group and no treatment group (RR $1.35,95 \%$ CI 1.20 to 1.51$)$. Another RCT [17] did not find significant benefit in acupuncture group. Significant benefit was found in acupuncture plus moxibustion group in an RCT [15].

ITT analysis was performed on three trials, however, the effect size and direction of correction rate remained the same $[6,14,15]$.

Two CCTs [22,24] found significant benefit in moxibustion group and a CCT [22] showed significant benefit in acupuncture group (Table 2).

\section{Moxibustion, laser stimulation or ear acupuncture versus knee-chest position}

Three RCTs $[12,18,20]$ published in Chinese found no significant difference between moxibustion and knee-chest position (RR $1.30,95 \%$ CI 0.95 to 1.79 ), analyzed in a random effect model with significant heterogeneity $\left(\mathrm{I}^{2}=\right.$ $77.0 \%)$. However, a meta-analysis showed positive results (RR 1.33, 95\% CI 1.18 to1.50), analyzed in a fixed effect model.

Two CCTs $[21,26]$ published in Chinese showed significant benefit in moxibustion compared with knee-chest position, analyzed in a fixed effect model (RR 3.36, 95\% CI 1.87 to 6.05 ), while they showed no significant benefit, analyzed in a random effect model (RR 6.31, 95\% CI 0.63 to 63.17 , significant heterogeneity $\left.\mathrm{I}^{2}=77.7 \%\right)$. One CCT [27] on ear acupuncture and knee-chest position found significant benefit in ear acupuncture group.

Two CCTs $[23,25]$ on laser stimulation found significant benefit compared with knee-chest position (Table 2). 


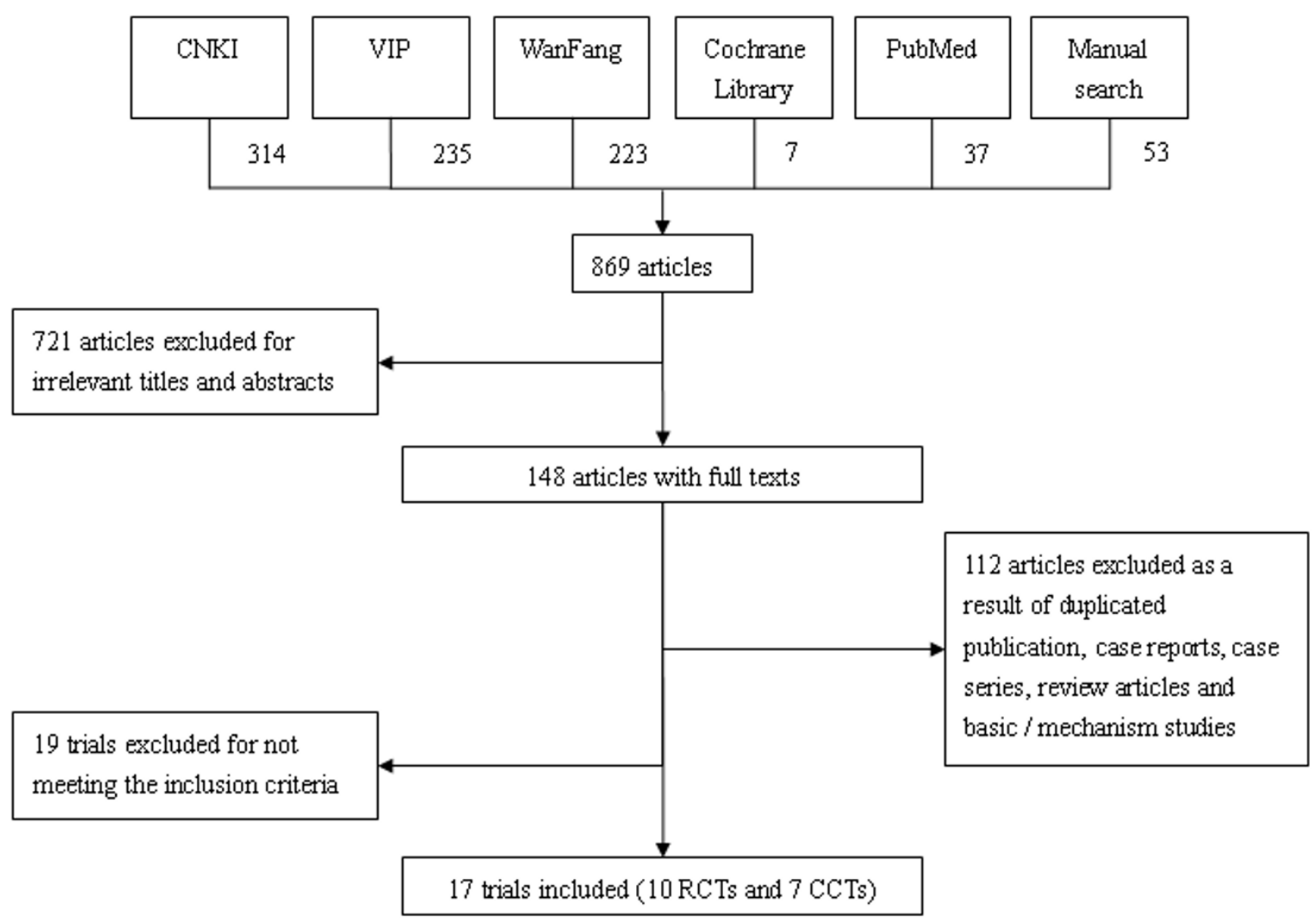

\section{Figure I}

Process of trial identification and selection.

Moxibustion plus other interventions versus other interventions An RCT [16], which investigated moxibustion on Zhiyin plus raising buttocks method versus raising buttocks method alone, showed significant benefit in the combination treatment group. Another RCT [13], which investigated moxibustion on Zhiyin plus knee-chest position versus knee-chest position alone, showed significant benefit in the combination treatment group. A meta-analysis of the two RCTs showed significant benefit in favour of the combination treatment. A CCT [23] compared moxibustion plus raising buttocks method with raising buttocks method alone but did not find significant difference between the two groups (Table 2).

Laser stimulation on Zhiyin versus other interventions

An RCT [23] on laser stimulation plus knee-chest position demonstrated significant benefit compared to pelvis rotating treatment which is an exercise to rotate a pregnant woman's pelvis for the correction of breech presentation.

A CCT [25] compared laser stimulation with raising buttocks method and showed significant benefit in laser stimulation group (Table 2).

\section{Publication biases}

Among the comparative trials, the maximal number of trials in one outcome was three. Due to the low number of trials, no meaningful funnel plots could be produced.

\section{Safety}

Four trials reported outcomes of adverse events unrelated to moxibustion treatment. Cardini and Weixin [6] reported two premature births and four preterm premature rupture of membranes (PPROM) in the treatment group among 129 participants, while three premature births, one intrauterine foetal death and 12 PPROM were reported in the control group. Cardini et al. [14] reported two cases of preterm deliveries, one of which was due to PPROM.

\section{Discussion}

From the findings of the present study, moxibustion, acupuncture and other acupoint stimulation appear to be effective in the correction of breech presentation. However, the number of available trials was insufficient for us to draw a confident conclusion. 
Table I: Characteristics of included trials of moxibustion, acupuncture and other acupoint stimulation for breech presentation

\begin{tabular}{|c|c|c|c|c|c|c|c|}
\hline Study ID & Design & Sample size & $\begin{array}{c}\text { Age (year) } \\
\text { (Rx/control) }\end{array}$ & $\begin{array}{c}\text { Gestation age } \\
\text { (week) } \\
\text { (Rx/control) }\end{array}$ & Intervention & Control & Outcomes \\
\hline Cardini 2005 [14] & RCT & 123 & 31 & 33 & $\begin{array}{l}\text { moxibustion on } \\
\text { Zhiyin }\end{array}$ & no treatment & $\begin{array}{l}\text { cephalic } \\
\text { presentation }\end{array}$ \\
\hline Cardini 1998 [6] & RCT & 260 & $25.5 / 25.2$ & $33 / 33$ & $\begin{array}{l}\text { moxibustion on } \\
\text { Zhiyin }\end{array}$ & no treatment & $\begin{array}{l}\text { number of } \\
\text { cephalic } \\
\text { presentation, } \\
\text { foetal activity; } \\
\text { number and } \\
\text { causes of } \\
\text { caesarean } \\
\text { deliveries, } \\
\text { spontaneous and } \\
\text { induced vaginal } \\
\text { deliveries, Apgar } \\
\text { score, adverse } \\
\text { events }\end{array}$ \\
\hline Huang 1990 [20] & $\mathrm{RCT}$ & 587 & NA & $28-32$ & $\begin{array}{l}\text { moxibustion on } \\
\text { Zhiyin }\end{array}$ & $\begin{array}{l}\text { no treatment or } \\
\text { knee-chest } \\
\text { position }\end{array}$ & $\begin{array}{l}\text { cephalic } \\
\text { presentation }\end{array}$ \\
\hline Yang 2006 [13] & $\mathrm{RCT}$ & 206 & $23.1-30.3$ & $28-34$ & $\begin{array}{l}\text { moxibustion on } \\
\text { Zhiyin plus knee- } \\
\text { chest position }\end{array}$ & $\begin{array}{l}\text { knee-chest } \\
\text { position }\end{array}$ & $\begin{array}{l}\text { cephalic } \\
\text { presentation, } \\
\text { adverse events }\end{array}$ \\
\hline Lin 2002 [18] & RCT & 122 & $21-38$ & $30-37$ & $\begin{array}{l}\text { moxibustion on } \\
\text { Zhiyin }\end{array}$ & $\begin{array}{l}\text { knee-chest } \\
\text { position }\end{array}$ & $\begin{array}{l}\text { cephalic } \\
\text { presentation }\end{array}$ \\
\hline Peng 2006 [12] & RCT & 80 & $21-36$ & $30-34$ & $\begin{array}{l}\text { moxibustion on } \\
\text { Zhiyin }\end{array}$ & $\begin{array}{l}\text { knee-chest } \\
\text { position }\end{array}$ & $\begin{array}{l}\text { cephalic } \\
\text { presentation }\end{array}$ \\
\hline Chen 2004 [16] & RCT & 142 & $22-38 / 22-39$ & $30-34 / 30-34$ & $\begin{array}{l}\text { moxibustion on } \\
\text { Zhiyin plus raising } \\
\text { buttocks method }\end{array}$ & $\begin{array}{l}\text { raising buttocks } \\
\text { method }\end{array}$ & $\begin{array}{l}\text { cephalic } \\
\text { presentation }\end{array}$ \\
\hline Habek 2003 [17] & RCT & 67 & $22 \pm 3.1 / 23 \pm 1.3$ & $34-37$ & $\begin{array}{l}\text { acupuncture on } \\
\text { Zhiyin }\end{array}$ & no treatment & $\begin{array}{l}\text { cephalic } \\
\text { presentation }\end{array}$ \\
\hline Neri 2004 [15] & RCT & 240 & $31.7+4.7 / 30.1+3.6$ & $33.5+0.6 / 33.7+0.7$ & $\begin{array}{l}\text { acupuncture plus } \\
\text { moxibustion on } \\
\text { Zhiyin }\end{array}$ & no treatment & $\begin{array}{l}\text { cephalic } \\
\text { presentation, } \\
\text { adverse events }\end{array}$ \\
\hline Ye 1998 [19] & RCT & 263 & 28.35 & $28-36 / 28-33$ & $\begin{array}{l}\text { laser stimulation } \\
\text { on Zhiyin }\end{array}$ & $\begin{array}{l}\text { knee-chest } \\
\text { position plus } \\
\text { pelvis rotating }\end{array}$ & $\begin{array}{l}\text { cephalic } \\
\text { presentation }\end{array}$ \\
\hline Liang 2004 [2I] & ССТ & 320 & NA & 28 & $\begin{array}{l}\text { moxibustion on } \\
\text { Zhiyin }\end{array}$ & $\begin{array}{l}\text { knee-chest } \\
\text { position }\end{array}$ & $\begin{array}{l}\text { cephalic } \\
\text { presentation }\end{array}$ \\
\hline Xiong 1991 [26] & СCT & 60 & $20-28 / 20-28$ & $32-36 / 32-36$ & $\begin{array}{l}\text { moxibustion on } \\
\text { Zhiyin }\end{array}$ & $\begin{array}{l}\text { knee-chest } \\
\text { position }\end{array}$ & $\begin{array}{l}\text { cephalic } \\
\text { presentation }\end{array}$ \\
\hline Wu 1995 [23] & CCT & 820 & $20-37$ & $20-37$ & $\begin{array}{l}\text { moxibustion on } \\
\text { Zhiyin plus raising } \\
\text { buttocks method } \\
\text { or laser } \\
\text { stimulation on } \\
\text { Zhiyin }\end{array}$ & $\begin{array}{l}\text { raising buttocks } \\
\text { method or knee- } \\
\text { chest position }\end{array}$ & $\begin{array}{l}\text { cephalic } \\
\text { presentation }\end{array}$ \\
\hline Jiang 1993 [25] & CCT & 382 & $20-38$ & $30-40$ & $\begin{array}{l}\text { laser stimulation } \\
\text { on Zhiyin }\end{array}$ & $\begin{array}{l}\text { knee-chest } \\
\text { position }\end{array}$ & $\begin{array}{l}\text { cephalic } \\
\text { presentation }\end{array}$ \\
\hline Qin 1989 [27] & CCT & 150 & NA & $30-37$ & ear acupuncture & $\begin{array}{l}\text { knee-chest } \\
\text { position }\end{array}$ & $\begin{array}{l}\text { cephalic } \\
\text { presentation }\end{array}$ \\
\hline $\begin{array}{l}\text { Kanakura 200I } \\
\text { [22] }\end{array}$ & ССТ & 548 & 28.4 & 28 (minimal) & $\begin{array}{l}\text { moxibustion or } \\
\text { electro- } \\
\text { acupuncture }\end{array}$ & no treatment & $\begin{array}{l}\text { cephalic } \\
\text { presentation }\end{array}$ \\
\hline Cardini 1993 [24] & ССТ & 41 & $20-37$ & $22-31$ & $\begin{array}{l}\text { moxibustion on } \\
\text { Zhiyin }\end{array}$ & no treatment & $\begin{array}{l}\text { cephalic } \\
\text { presentation }\end{array}$ \\
\hline
\end{tabular}


Table 2: Efficacy of moxibustion, acupuncture or other acupoint stimulations for the correction of breech presentation

\begin{tabular}{|c|c|c|c|c|}
\hline & $\begin{array}{l}\text { Treatment } \\
(\mathrm{n} / \mathrm{N}, \%)\end{array}$ & $\begin{array}{l}\text { Control } \\
(\mathrm{n} / \mathrm{N}, \%)\end{array}$ & $\begin{array}{l}\text { Relative benefit } \\
(95 \% \mathrm{Cl})\end{array}$ & $P$ value \\
\hline \multicolumn{5}{|c|}{ Randomized controlled trial } \\
\hline \multicolumn{5}{|c|}{ Moxibustion on Zhiyin vs no treatment } \\
\hline Cardini $1998[6]$ & $98 / 129(76.0)$ & $62 / 106(58.5)$ & $1.30[1.08,1.57]$ & 0.006 \\
\hline Huang $1990[20]$ & $150 / 193$ (77.7) & $106 / 200(53.0)$ & $1.47[1.26,1.71]$ & $<0.00001$ \\
\hline Cardini 2005 [14] & $22 / 65(33.8)$ & $21 / 58(36.2)$ & $0.93[0.58,1.51]$ & 0.78 \\
\hline Meta-analysis & $270 / 387(69.8)$ & $189 / 364(51.9)$ & $1.35[1.20,1.51]$ & $<0.00001$ \\
\hline \multicolumn{5}{|c|}{ Acupuncture on Zhiyin vs no treatment } \\
\hline Habek 2003 [17] & $3 \mid / 34(9 \mid .2)$ & $26 / 33(78.8)$ & $1.16[0.94,1.42]$ & 0.16 \\
\hline \multicolumn{5}{|c|}{ Acupuncture plus moxibustion on Zhiyin vs no treatment } \\
\hline Neri 2004 [15] & $61 / / 20(50.8)$ & $43 / 120(35.8)$ & $1.42[1.05,1.91]$ & 0.02 \\
\hline \multicolumn{5}{|c|}{ Moxibustion on Zhiyin vs knee-chest position } \\
\hline Huang $1990[20]$ & $150 / 193$ (77.7) & $115 / 194(59.3)$ & $1.31[1.14, \mid .51]$ & 0.0001 \\
\hline Lin 2002 [18] & $58 / 63(92.1)$ & $31 / 59(52.5)$ & $1.75[1.36,2.26]$ & $<0.0001$ \\
\hline Peng 2006 [12] & $16 / 40(40.0)$ & $20 / 40(50.0)$ & $0.80[0.49,1.31]$ & 0.73 \\
\hline Meta-analysis* & $224 / 296(75.7)$ & $166 / 293(56.7)$ & $1.30[0.95,1.79]$ & 0.1 \\
\hline \multicolumn{5}{|c|}{ Moxibustion on Zhiyin plus raising buttocks method vs raising buttocks method } \\
\hline Chen 2004 [16] & $67 / 73(91.8)$ & $36 / 69(52.2)$ & $1.76[1.39,2.23]$ & 0.02 \\
\hline \multicolumn{5}{|c|}{ Moxibustion on Zhiyin plus knee-chest position vs knee-chest position } \\
\hline Yang 2006 [13] & $90 / 103(87.4)$ & $77 / 103(74.8)$ & $1.17[1.02,1.34]$ & $<0.00001$ \\
\hline Meta-analysis & $157 / 176(89.2)$ & $113 / 172(65.7)$ & $1.36[1.21,1.54]$ & $<0.00001$ \\
\hline \multicolumn{5}{|c|}{ Laser stimulation on Zhiyin vs knee-chest position plus pelvis rotating } \\
\hline Ye $1998[19]$ & $108 / 133(81.2)$ & $73 / 130(56.2)$ & $1.45[1.22,1.72]$ & $<0.0001$ \\
\hline \multicolumn{5}{|c|}{ Controlled clinical trial } \\
\hline \multicolumn{5}{|c|}{ Moxibustion on Zhiyin vs no treatment } \\
\hline Kanakura 200I [22] & $123 / 133(92.5)$ & $165 / 224(73.7)$ & $1.26[1.15,1.38]$ & $<0.00001$ \\
\hline Cardini $1993[24]$ & $16 / 23(69.6)$ & $7 / 18(38.9)$ & $1.79[0.94,3.39]$ & 0.07 \\
\hline Meta-analysis & $139 / 156(89.1)$ & I72/242 (7I.I) & $1.29[1.17,1.42]$ & $<0.00001$ \\
\hline \multicolumn{5}{|c|}{ Acupuncture on Zhiyin vs no treatment } \\
\hline Kanakura 200I [22] & $|7| /|9|(89.5)$ & $160 / 217(73.7)$ & $1.21[1.11,1.33]$ & $<0.001$ \\
\hline \multicolumn{5}{|c|}{ Moxibustion on Zhiyin vs knee-chest position } \\
\hline Liang 2004 [2I] & |44//60 (90.0) & $126 / 160(78.8)$ & $1.14[1.04,1.26]$ & 0.007 \\
\hline Xiong 1991 [26] & $29 / 30(96.7)$ & $16 / 30(53.3)$ & $1.81[1.29,2.55]$ & 0.003 \\
\hline Meta-analysis* & $173 / 190(91.1)$ & $142 / 190(74.7)$ & $6.31[0.63,63.17]$ & 0.12 \\
\hline \multicolumn{5}{|c|}{ Laser stimulation on Zhiyin vs knee-chest position } \\
\hline Jiang YH 1993 [25] & $218 / 278(78.4)$ & $66 / 104(63.5)$ & $1.24[1.05,1.45]$ & 0.003 \\
\hline Wu 1995 [23] & $314 / 432(72.7)$ & $25 / 51(49.0)$ & $1.48[1.11,1.97]$ & 0.0007 \\
\hline Meta-analysis & $532 / 710(74.9)$ & $91 / 155(58.7)$ & $1.30[1.10,1.54]$ & $<0.00001$ \\
\hline \multicolumn{5}{|c|}{ Ear acupuncture vs knee-chest position } \\
\hline Qin 1989 [27] & $84 / 99(84.8)$ & $26 / 39(66.7)$ & $1.27[1.00,1.61]$ & 0.02 \\
\hline \multicolumn{5}{|c|}{ Moxibustion on Zhiyin plus raising buttocks method vs raising buttocks method } \\
\hline Wu 1995 [23] & $103 / 192(53.6)$ & $76 / 145(52.4)$ & $1.02[0.84,1.25]$ & 0.82 \\
\hline \multicolumn{5}{|c|}{ Laser stimulation on Zhiyin vs raising buttocks method } \\
\hline Wu 1995 [23] & $314 / 432(72.7)$ & $76 / 145(52.4)$ & $1.39[1.18,1.64]$ & 0.00001 \\
\hline
\end{tabular}

\footnotetext{
*Random effect model
} 
In both RCTs and CCTs, moxibustion showed significant favourable differences in comparison with no treatment. However, meta-analysis of both the RCTs and CCTs comparing moxibustion with knee-chest position showed non-significant differences in a random effect model due to a highly heterogeneity. The results were positive in a fixed effect model, which should be interpreted with caution.

To investigate the efficacy of knee-chest position in comparison with no treatment or placebo, we searched PubMed and identified a Cochrane systematic review of three RCTs [28]. This review did not find adequate evidence to support that moxibustion or knee-chest position had significant benefits in comparison with no treatment. However, our findings agree with a recently published systematic review of six RCTs and three cohort studies [29] suggesting that moxibustion and other acupuncture-type interventions at acupoint BL67 are effective in the correction of breech presentation and that the methodological quality of the available trials was limited. An ongoing multi-centre randomized trial may provide further evidence for the efficacy [30].

No biological synergistic actions have been suggested between moxibustion and other interventions such as knee-chest position or raising buttocks method; thus, these interventions may be independent from each other. When moxibustion plus another intervention shows significant beneficial effects compared with the respective non-moxibustion intervention, we may assume that the differences are caused by moxibustion. Under this assumption, we combined the trials with similar study designs $[13,16]$ in our meta-analysis.

We included in the present review both randomized and non-randomized trials because many trials carried out in China are non-randomized which may provide supplementary evidence to randomized trials [31].

Double blinding was not practised in these trials as it is not practical to mask the practitioners and/or the patients during moxibustion and other acupoint stimulation interventions lack a suitable placebo. Outcomes for the correction of breech presentation were determined objectively by ultrasound.

The effectiveness of moxibustion may vary depending on participants' culture background, belief, preference and expectation as evidenced in two RCTs $[6,14]$. It should also be noted that breech presentation was corrected spontaneously at about $50 \%$ in the non treatment groups. Thus, these factors should be taken into consideration in designing clinical trials. Incorporating qualitative research into clinical trials may help interpret research findings [32].
Further randomized trials are warranted, in which several aspects should be addressed, such as study settings, patient preferences and expectations (qualitative research), characteristics of the pregnant women (e.g. age, ethnic group, term of pregnancy), a consensus protocol of the intervention, and clinical and end-point outcomes. Trials should be reported according to the CONSORT Statement [33].

\section{Conclusion}

From the findings of the present study, moxibustion, acupuncture and laser stimulation at acupoints showed beneficial effects for the correction of breech presentation. However, studies such as multi-centre trials are warranted.

\section{Abbreviations}

CCTs: controlled clinical trials; CI: confidence interval; CNKI: China National Knowledge Infrastructure; $\mathrm{I}^{2}$ : I square; ITT: intention to treat analysis; PPROM: preterm premature rupture of membranes; RCTs: randomized controlled trials; RR: risk ratio; VIP: Chinese Scientific Journal Database; WanFang: WanFang Database.

\section{Competing interests}

The authors declare that they have no competing interests.

\section{Authors' contributions}

$\mathrm{XL}$ and JPL conceived the review topic and drafted the manuscript. JPL revised the manuscript and provided perspectives on methodological issues. JH and XL performed the electronic and manual searches respectively, and conducted study selection, data extraction and analysis, and quality assessment. XYW and HRZ performed the manual searches, data extraction and quality assessment. All authors read and approved the final version of the manuscript.

\section{Additional material}

\section{Additional file 1}

Clinical trials excluded from the present review. The table provides the bibliographic information of the clinical trials excluded from the present review and the reasons for exclusion.

Click here for file

[http://www.biomedcentral.com/content/supplementary/1749-

8546-4-4-S1.doc]

\section{Acknowledgements}

The work of XL and JPL was supported by a grant (2006CB504602) from the National Basic Research Program of China (i.e. '973' Program) and the 'I I I' Project (B08006). The work of JPL was also partially supported by a grant (R24 AT001293) from the National Center for Complementary and Alternative Medicine (NCCAM), US National Institutes of Health (NIH). 


\section{References}

I. Cruickshank DP: Breech presentation. Clin Obstet Gynecol 1986, 29:255-263.

2. Roberts CL, Algert CS, Peat B, Henderson-Smart D: Small fetal size: a risk factor for breech birth at term. Int J Gynaecol Obstet 1999, 67:1-18

3. Irion $O$, Hirsbrunner Almagbaly $P$, Morabia $A$ : Planned vaginal delivery versus elective caesarean section: a study of 705 singleton term breech presentations. Br J Obstet Gynaecol 1998, 105:710-717.

4. Boog G: Alternative methods instead of external cephalic version in the event of breech presentation. Review of the literature. J Gynecol Obstet Biol Reprod (Paris) 2004, 33:94-98.

5. Lavender T, Hofmeyr GJ, Neilson JP, Kingdon C, Gyte GML: Caesarean section for non-medical reasons at term. Cochrane Database of Systematic Reviews 2006:Art. No.: CD004660. DOI: 10.1002 |465 I 858.CD004660.pub2

6. Cardini F, Weixin $\mathrm{H}$ : Moxibustion for correction of breech presentation: a randomized controlled trial. JAMA 1998, 280: I580- I584.

7. Higgins JPT, Green S, (Eds): Cochrane Handbook for Systematic Reviews of Interventions Version 5.0.0 2008 [http://www.cochrane-hand book.org].

8. Altman DG, Schulz KF, Moher D, Egger M, Davidoff F, Elbourne D, Gøtzsche PC, Lang T, CONSORT GROUP (Consolidated Standards of Reporting Trials): The revised CONSORT statement for reporting randomized trials: explanation and elaboration. Ann Intern Med 200 I, I34:663-694.

9. Moher D, Schulz KF, Altman D: The CONSORT statement: revised recommendations for improving the quality of reports of parallel-group randomized trials. JAMA 200I, 285:1987-1991.

10. Ip S, Chung M, Raman G, Chew P, Magula N, DeVine D, Trikalinos T, Lau J: Breastfeeding and Maternal and Infant Health Outcomes in Developed Countries. In Evidence Report/Technology Assessment No. 153 (Prepared by Tufts-New England Medical Center Evidence-based Practice Center, under Contract No. 290-02-0022). AHRQ Publication No. 07-E007 Rockville, MD: Agency for Healthcare Research and Quality; 2007.

I I. Higgins JPT, Green S, (Eds): 9.5.2 Identifying and measuring heterogeneity. Cochrane Handbook for Systematic Reviews of Interventions Version 5.0.I 2008 [http://www.cochrane-handbook.org]. The Cochrane Collaboration

12. Peng SM: Comparison of several methods for conversion of breech presentation. Zhongguo Yiyao Luntan 2006, 4:30.

13. Yang FQ: Comparison of knee-chest position plus moxibustion on Zhiyin with knee-chest position for breech presentation. Sichuan Zhongyi 2006, 24:106-107.

14. Cardini F, Lombardo P, Regalia AL, Regaldo G, Zanini A, Negri MG Panepuccia $L$, Todros $T$ : A randomized controlled trial of moxibustion for breech presentation. BJOG 2005, I I 2:743-747.

15. Neri I, Airola G, Contu G, Allais G, Facchinetti F, Benedetto C: Acupuncture plus moxibustion to resolve breech presentation: a randomized controlled study. J Matern Fetal Neonatal Med 2004, I 5:247-252

16. Chen Y, Yang LW: Moxibustion on Zhiyin plus raising buttocks in a lateral position for correction fetal presentation in $\mathbf{7 3}$ cases. Zhongyiyao Linchuang Zazhi 2004, I 6:333.

17. Habek D, Cerkez Habek J, Jagust M: Acupuncture Conversion of Fetal Breech Presentation. Fetal Diagn Ther 2003, I 8:4I8-42I.

18. Lin YP, Zhang DQ, Hao YQ, Duan XW: Combination of $\mathbf{M}$ oxibustion at Point Zhiyin and Knee-Chest Position for Correction of Breech pregnancy in 63 Cases. Zhongguo Zhenjiu 2002, 22:8I|-8I2.

19. Ye LL, Zhu YP: He-Ne laser stimuliation for correcting breech presentaion in I33 cases. Xiandai Fuchanke Jinzhan 1998, 7:379.

20. Huang WX: Comparison study on moxibustion and kneechest position for conversion of fetal presentation. Zhongguo Zhongxiyi Jiehe Zazhi 1990, I 0:105-106.

21. Liang JL, Chen SR, Li YP: Comparative Analysis of Moxibustion at Zlliyin Acupoint and Knee-chest Posture in Correcting Breech Presentation, Report of $\mathbf{3 2 0}$ Cases. Huaxia Yiyao 2004, I 7:I I-12.

22. Kanakura Y, Kometani K, Nagata T, Niwa K, Kamatsuki H, Shinzato $\mathrm{Y}$, Tokunaga $\mathrm{Y}$ : Moxibustion treatment of breech presentation. Am J Chin Med 200I, 29:37-45.
23. $\mathrm{Wu}$ JR: He-Ne laser for correcting breech presentation in $\mathbf{4 3 2}$ cases. Xiandai Fuchanke Jinzhan 1995, 4: I40-I4I.

24. Cardini F, Marcolongo A: Moxibustion for correction of breech presentation: a clinical study with retrospective control. Am J Chin Med 1993, 2 I:133-138.

25. Jiang $\mathrm{YH}$ : Laser needle stimulation on Zhiyin for convertion of breech presentation in 278 cases. Hunan Yixue 1993, 1 0:67.

26. Xiong $\mathrm{CH}$ : Comparative observation on moxibustion on Zhiyin with knee-chest position for abnormal fetal position in $\mathbf{3 0}$ cases. Hunan Zhongyi Zazhi 1991, 6:23-24.

27. Qin GF, Tang HJ: Ear pressing for abnormal fetal presentation in 413 cases. Zhongyi Zazhi 1989, 30:30-32.

28. Coyle ME, Smith CA, Peat B: Cephalic version by moxibustion for breech presentation. Cochrane Database Syst Rev 2005. Art

29. Berg I Van den, Bosch JL, Jacobs B, Bouman I, Duvekot J], Hunink MG: Effectiveness of acupuncture-type interventions versus expectant management to correct breech presentation: A systematic review. Complement Ther Med 2008, 16:92-100.

30. Vas J, Aranda JM, Barón M, Perea-Milla E, Méndez C, Ramírez C, Aguilar I, Modesto M, Lara AM, Martos F, García-Ruiz AJ: Correcting non cephalic presentation with moxibustion: study protocol for a multi-centre randomised controlled trial in general practice. BMC Complement Altern Med 2008, 8:22.

31. Concato J, Shah N, Horwitz RI: Randomized, controlled trials, observational studies and the hierarchy of research designs [J]. N Engl J Med 2000, 342: |887-1892.

32. Mitchell M, Allen K: An exploratory study of women's experiences and key stakeholders views of moxibustion for cephalic version in breech presentation. Complement Ther Clin Pract 2008, I 4(4):264-72.

33. The CONSORT Statement [http://www.consort-statement.org]
Publish with Biomed Central and every scientist can read your work free of charge

"BioMed Central will be the most significant development for disseminating the results of biomedical research in our lifetime. "

Sir Paul Nurse, Cancer Research UK

Your research papers will be:

- available free of charge to the entire biomedical community

- peer reviewed and published immediately upon acceptance

- cited in PubMed and archived on PubMed Central

- yours - you keep the copyright
BioMedcentral 